\title{
RESOLUTION OF SMOOTH GROUP ACTIONS
}

\author{
PIERRE ALBIN AND RICHARD MELROSE
}

\begin{abstract}
A refined form of the 'Folk Theorem' that a smooth action by a compact Lie group can be (canonically) resolved, by iterated blow up, to have unique isotropy type is proved in the context of manifolds with corners. This procedure is shown to capture the simultaneous resolution of all isotropy types in a 'resolution structure' consisting of equivariant iterated fibrations of the boundary faces. This structure projects to give a similar resolution structure for the quotient. In particular these results apply to give a canonical resolution of the radial compactification, to a ball, of any finite dimensional representation of a compact Lie group; such resolutions of the normal action of the isotropy groups appear in the boundary fibers in the general case.
\end{abstract}

\section{CONTENTS}

Introduction

1. Manifolds with corners

2. Blow up

3. Resolution structures

4. Group actions

6. Boundary resolution 17

7. Resolution of $G$-actions 19

8. Resolution of orbit spaces 21

9. Equivariant maps and resolution 22

References

\section{INTRODUCTION}

Borel showed that if the isotropy groups of a smooth action by a compact Lie group, $G$, on a compact manifold, $M$, are all conjugate then the orbit space, $G \backslash M$, is smooth. Equivariant objects on $M$, for such an action, can then be understood directly as objects on the quotient. In the case of a free action, which is to say a principal $G$-bundle, Borel showed that the equivariant cohomology of $M$ is then naturally isomorphic to the cohomology of $G \backslash M$. In a companion paper, [1, this is extended to the unique isotropy case to show that the equivariant cohomology of $M$ reduces to the cohomology of $G \backslash M$ with coefficients in a flat bundle (the Borel bundle). In this paper we show how, by resolution, a general smooth compact group action on a compact manifold is related to an action with unique isotropy type on a

The first author was partially supported by an NSF postdoctoral fellowship and NSF grant DMS-0635607002 and the second author received partial support under NSF grant DMS-1005944. 
resolution, canonically associated to the given action, of the manifold to a compact manifold with corners.

The resolution of a smooth Lie group action is discussed by Duistermaat and Kolk [7. (which we follow quite closely), by Kawakubo [1] and by Wasserman [13. but goes back at least as far as Jänich [10, Hsiang [9], and Davis 6]. See also the discussion by Brüning, Kamber and Richardson [5] which appeared after the present work was complete. In these approaches there are either residual finite group actions, particularly reflections, as a consequence of the use of real projective blow up or else the manifold is repeatedly doubled. Using radial blow up, and hence working in the category of manifolds with corners, such problems do not arise.

For a general group action, $M$ splits into various isotropy types

$$
M^{[K]}=\left\{\zeta \in M: G_{\zeta} \text { is conjugate to } K\right\}, G_{\zeta}=\{g \in G: g \zeta=\zeta\}, \zeta \in M .
$$

These are smooth manifolds but not necessarily closed and the orbit space is then in general singular. We show below that each $M^{[K]}$ has a natural compactification to a manifold with corners, $Y_{[K]}$, the boundary hypersurfaces of which carry equivariant fibrations with bases the compactifications of the isotropy types contained in the closure of $M^{[K]}$ and so corresponding to larger isotropy groups. Each fiber of these fibrations is the canonical resolution of the normal action of the larger isotropy group. These fibrations collectively give what we term a resolution structure, $\left\{\left(Y_{I}, \phi_{I}\right) ; I \in \mathcal{I}\right\}$, the index set being the collection of conjugacy classes of isotropy groups, i.e. of isotropy types, of the action. If $M$ is connected there is always a minimal 'open' isotropy type $\mu \in \mathcal{I}$, for which the corresponding manifold, $Y_{\mu}=Y(M)$, (possibly not connected) gives a resolution of the action on $M$. That is, there is a smooth $G$-action on $Y(M)$ with unique isotropy type and a smooth $G$-equivariant map

$$
\beta: Y(M) \longrightarrow M
$$

which is a diffeomorphism of the interior of $Y(M)$ to the minimal isotropy type. Here, $\beta$ is the iterated blow-down map for the resolution. There is a $G$-invariant partition of the boundary hypersurfaces of $Y(M)$ into non-self-intersecting collections $H_{I}$, labelled by the non-minimal isotropy types $I \in \mathcal{I} \backslash\{\mu\}$, and carrying $G$-equivariant fibrations

$$
\phi_{I}: H_{I} \longrightarrow Y_{I} .
$$

Here $Y_{I}$ resolves the space $M_{I}$, the closure of the corresponding isotropy type $M^{I}$,

$$
\beta_{I}: Y_{I} \longrightarrow M_{I},\left.\beta\right|_{H_{I}}=\beta_{I} \circ \phi_{I}
$$

Thus the inclusion relation between the $M_{I}$ corresponding to the stratification of $M$ by isotropy types, is 'resolved' into the intersection relation between the $H_{I}$. The resolution structure for $M$, thought of as the partition of the boundary hypersurfaces with each collection carrying a fibration, naturally induces a resolution structure for each $Y_{I}$. Since the fibrations are equivariant the quotients $Z_{I}$ of the $Y_{I}$ by the group action induce a similar resolution structure on the quotient $Z(M)$ of $Y(M)$ which resolves the quotient, the orbit space, $G \backslash M$.

As noted above, in a companion paper [1], various cohomological consequences of this construction are derived. The 'lifts' of both the equivariant cohomology and equivariant K-theory of a manifold with a group action to its resolution structure are described. These lifted descriptions then project to corresponding realizations 
of these theories on the resolution structure for the quotient. As a consequence of the forms of these resolved and projected theories a 'delocalized' equivariant cohomology is defined, and shown to reduce to the cohomology of Baum, Brylinski and MacPherson in the Abelian case in 3 . The equivariant Chern character is then obtained from the usual Chern character by twisting with flat coefficients and establishes an isomorphism between equivariant K-theory with complex coefficients and delocalized equivariant cohomology. Applications to equivariant index theory will be described in [2].

For the convenience of the reader a limited amount of background information on manifolds with corners and blow up is included in the first two sections. The abstract notion of a resolution structure on a manifold with corners is discussed in $\$ 3$ and the basic properties of G-actions on manifolds with corners are described in \$4 The standard results on tubes and collars are extended to this case in 95 . In \$6 it is shown that for a general action the induced action on the set of boundary hypersurfaces can be appropriately resolved. The canonical resolution itself is then presented in \$7, including some simple examples, and the induced resolution of the orbit space is considered in 8 . Finally 99 describes the resolution of an equivariant embedding and the 'relative' resolution of the total space of an equivariant fibration.

The authors are grateful to Eckhard Meinrenken for very helpful comments on the structure of group actions, and to an anonymous referee for remarks improving the exposition.

\section{MANiFOLDS WiTH CORNERS}

By a manifold with corners, $M$, we shall mean a topological manifold with boundary with a covering by coordinate charts

$$
M=\bigcup_{j} U_{j}, F_{j}: U_{j} \longrightarrow U_{j}^{\prime} \subset \mathbb{R}^{m, \ell}=[0, \infty)^{\ell} \times \mathbb{R}^{m-\ell},
$$

where the $U_{j}$ and $U_{j}^{\prime}$ are (relatively) open, the $F_{j}$ are homeomorphisms and the transition maps

$$
F_{i j}: F_{i}\left(U_{i} \cap U_{j}\right) \longrightarrow F_{j}\left(U_{i} \cap U_{j}\right), U_{i} \cap U_{j} \neq \emptyset
$$

are required to be smooth in the sense that all derivatives are bounded on compact subsets; an additional condition is imposed below. The ring of smooth functions $\mathcal{C}^{\infty}(M) \subset \mathcal{C}^{0}(M)$ is fixed by requiring $\left(F_{j}^{-1}\right)^{*}\left(\left.u\right|_{U_{j}}\right)$ to be smooth on $U_{j}^{\prime}$, in the sense that it is the restriction to $U_{j}^{\prime}$ of a smooth function on an open subset of $\mathbb{R}^{m}$.

The part of the boundary of smooth codimension one, which is the union of the inverse images under the $F_{i}$ of the corresponding parts of the boundary of the $\mathbb{R}^{m, \ell}$, is dense in the boundary and the closure of each of its components is a boundary hypersurface of $M$. More generally we shall call a finite union of non-intersecting boundary hypersurfaces a collective boundary hypersurface. We shall insist, as part of the definition of a manifold with corners, that these boundary hypersurfaces each be embedded, meaning near each point of each of these closed sets, the set itself is given by the vanishing of a local smooth defining function $x$ which is otherwise positive and has non-vanishing differential at the point. In the absence of this condition $M$ is a tied manifold. It follows that each collective boundary hypersurface, $H$, of a manifold with corners is globally the zero set of a smooth, otherwise positive, boundary defining function $\rho_{H} \in \mathcal{C}^{\infty}(M)$ with differential non-zero on $H$; conversely $H$ determines $\rho_{H}$ up to a positive smooth multiple. The set of connected 
boundary hypersurfaces is denoted $\mathcal{M}_{1}(M)$ and the boundary faces of $M$ are the components of the intersections of elements of $\mathcal{M}_{1}(M)$. We denote by $\mathcal{M}_{k}(M)$ the set of boundary faces of codimension $k$. Thus if $F \in \mathcal{M}_{k}(M)$ and $F^{\prime} \in \mathcal{M}_{k^{\prime}}(M)$ then $F \cap F^{\prime}$ can be identified with the union over the elements of a subset (possibly empty of course) which we may denote $F \cap F^{\prime} \subset \mathcal{M}_{k+k^{\prime}}(M)$. Once again it is convenient to call a subset of $\mathcal{M}_{k}(M)$ with non-intersecting elements a collective boundary face, and then the collection of intersections of the elements of two collective boundary faces is a collective boundary face.
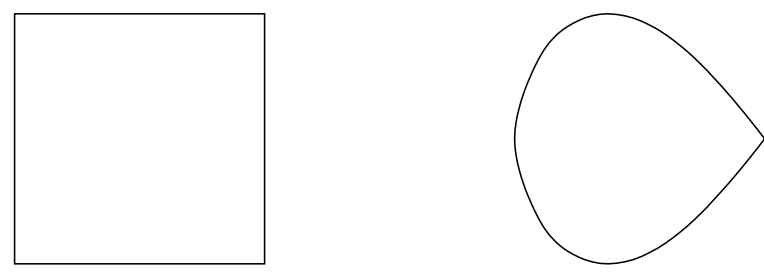

Figure 1. The square is a manifold with corners. The teardrop is only a tied manifold since its boundary hypersurface intersects itself.

By a manifold from now on we shall mean a manifold with corners, so the qualifier will be omitted except where emphasis seems appropriate. The traditional object will be called a boundaryless manifold.

As a consequence of the assumption that the boundary hypersurfaces are embedded, each boundary face of $M$ is itself a manifold with corners (for a tied manifold the boundary hypersurfaces are more general objects, namely articulated manifolds which have boundary faces identified). At each point of a manifold with corners there are, by definition, local product coordinates $x_{i} \geq 0, y_{j}$ where $1 \leq i \leq k$ and $1 \leq j \leq m-k$ (and either $k$ or $m-k$ can be zero) and the $x_{i}$ define the boundary hypersurfaces through the point. Unless otherwise stated, by local coordinates we mean local product coordinates in this sense. The local product structure near the boundary can be globalized:-

Definition 1.1. On a compact manifold with corners, $M$, a boundary product structure consists of a choice $\rho_{H} \in \mathcal{C}^{\infty}(M)$ for each $H \in \mathcal{M}_{1}(M)$, of a defining function for the each of the boundary hypersurfaces, an open neighborhood $U_{H} \subset M$ of each $H \in \mathcal{M}_{1}(M)$ and a smooth vector field $V_{H}$ defined in each $U_{H}$ such that

$$
\begin{gathered}
V_{H} \rho_{K}= \begin{cases}1 & \text { in } U_{H} \text { if } K=H \\
0 & \text { in } U_{H} \cap U_{K} \text { if } K \neq H,\end{cases} \\
{\left[V_{H}, V_{K}\right]=0 \text { in } U_{H} \cap U_{K} \forall H, K \in \mathcal{M}_{1}(M) .}
\end{gathered}
$$

Integration of each $V_{H}$ from $H$ gives a product decomposition of a neighborhood of $H$ as $\left[0, \epsilon_{H}\right] \times H, \epsilon_{H}>0$ in which $V_{H}$ is differentiation in the parameter space on which $\rho_{H}$ induces the coordinate. Shrinking $U_{H}$ allows it to be identified with such a neighborhood without changing the other properties (1.3). Scaling $\rho_{H}$ and $V_{H}$ allows the parameter range to be taken to be $[0,1]$ for each $H$.

Proposition 1.2. Every compact manifold has a boundary product structure. 
Proof. The construction of the neighborhoods $U_{H}$ and normal vector fields $V_{H}$ will be carried out inductively. For the inductive step it is convenient to consider a strengthened hypothesis. Note first that the data in (1.3) induces corresponding data on each boundary face $F$ of $M$ - where the hypersurfaces containing $F$ are dropped, and for the remaining hypersurfaces the neighborhoods are intersected with $F$ and the vector fields are restricted to $F$ - to which they are necessarily tangent. It may be necessary to subdivide the neighborhoods if the intersection $F \cap H$ has more than one component. In particular this gives data as in (1.3) but with $M$ replaced by $F$. So such data, with $M$ replaced by one of its hypersurfaces, induces data on all boundary faces of that hypersurface. Data as in (1.3) on a collection of boundary hypersurfaces of a manifold $M$, with the defining functions $\rho_{H}$ fixed, is said to be consistent if all restrictions to a given boundary face of $M$ are the same.

Now, let $\mathcal{B} \subset \mathcal{M}_{1}(M)$ be a collection of boundary hypersurfaces of a manifold $M$, on which boundary defining functions $\rho_{H}$ have been chosen for each $H \in \mathcal{M}_{1}(M)$, and suppose that neighborhoods $U_{K}$ and vector fields $V_{K}$ have been found satisfying (1.3) for all $K \in \mathcal{B}$. If $H \in \mathcal{M}_{1}(M) \backslash \mathcal{B}$ then we claim that there is a choice of $V_{H}$ and $U_{H}$ such that (1.3) holds for all boundary hypersurfaces in $\mathcal{B} \cup\{H\}$, with the neighborhoods possibly shrunk. To see this we again proceed inductively, by seeking $V_{H}$ only on the elements of a subset $\mathcal{B}^{\prime} \subset \mathcal{B}$ but consistent on all common boundary faces. The subset $\mathcal{B}^{\prime}$ can always be increased, since the addition of another element of $\mathcal{B} \backslash \mathcal{B}^{\prime}$ to $\mathcal{B}^{\prime}$ requires the same inductive step but in lower overall dimension, which we can assume already proved. Thus we may assume that $V_{H}$ has been constructed consistently on all elements of $\mathcal{B}$. Using the vector fields $V_{K}$, each of which is defined in the neighborhood $U_{K}$ of $K, V_{H}$ can be extended, locally uniquely, from the neighborhood of $K \cap H$ in $K$ on which it is defined to a neighborhood of $K \cap H$ in $M$ by demanding

$$
\mathcal{L}_{V_{K}} V_{H}=\left[V_{K}, V_{H}\right]=0 .
$$

The commutation condition and other identities follow from this and the fact that they hold on $K$. Moreover, the fact that the $V_{K}$ commute in the intersections of the $U_{K}$ means that these extensions of $V_{H}$ are consistent for different $K$ on their common domains. In this way $V_{H}$ satisfying all conditions in (1.3) has been constructed in a neighborhood of the part of the boundary of $H$ in $M$ corresponding to $\mathcal{B}$. In the complement of this part of the boundary one can certainly choose $V_{H}$ to satisfy $V_{H} \rho_{H}=1$ and combining these two choices using a partition of unity (with two elements) gives the desired additional vector field $V_{H}$ once the various neighborhoods $U_{K}$ are shrunk.

Thus, after a finite number of steps the commuting normal vector fields $V_{K}$ are constructed near each boundary hypersurface.

Note that this result is equally true if in the definition the set of boundary hypersurfaces is replaced with any partition into collective boundary hypersurfaces, however it is crucial that the different hypersurfaces in each collection do not intersect.

The existence of such normal neighborhoods of the boundary hypersurfaces ensures the existence of 'product-type' metrics. That is, one can choose a metric $g$ globally on $M$ which near each boundary hypersurface $H$ is of the form $d \rho_{H}^{2}+\phi_{H}^{*} h_{H}$ where $\phi_{H}: U_{H} \longrightarrow H$ is the projection along the integral curves of $V_{H}$ and $h_{H}$ is 
a metric, inductively of the same product-type, on $H$. Thus near a boundary face $F \in \mathcal{M}_{k}(M)$, which is defined by $\rho_{H_{i}}, i=1, \ldots, k$, the metric takes the form

$$
g=\sum_{i=1}^{k} d \rho_{H_{i}}^{2}+\phi_{F}^{*} h_{F}
$$

where $\phi_{F}$ is the local projection onto $F$ with leaves the integral surfaces of the $k$ commuting vector fields $V_{H_{i}}$. In particular

Corollary 1.3. On any manifold with corners there exists a metric g, smooth and non-degenerate up to all boundary faces, for which the boundary faces are each totally geodesic.

A diffeomorphism of a manifold sends boundary faces to boundary faces - which is to say there is an induced action on $\mathcal{M}_{1}(M)$.

Definition 1.4. A diffeomorphism $F$ of a manifold $M$ is said to be boundary intersection free if for each $H \in \mathcal{M}_{1}(M)$ either $F(H)=H$ or $F(H) \cap H=\emptyset$. More generally a collection $\mathcal{G}$ of diffeomorphisms is said to be boundary intersection free if $\mathcal{M}_{1}(M)$ can be partitioned into collective boundary hypersurfaces $B_{i} \subset \mathcal{M}_{1}(M)$, so the elements of each $B_{i}$ are disjoint, such that the induced action of each $F \in \mathcal{G}$ preserves the partition, i.e. maps each $B_{i}$ to itself.

A manifold with corners, $M$, can always be realized as an embedded submanifold of a boundaryless manifold. As shown in [12, if $\mathcal{F} \subset \mathcal{M}_{1}(M)$ is any disjoint collection of boundary hypersurfaces then the 'double' of $M$ across $\mathcal{F}$, meaning $2_{\mathcal{F}} M=M \sqcup M / \cup \mathcal{F}$ can be given (not however naturally) the structure of a smooth manifold with corners. If $\left\{\mathcal{F}_{1}, \ldots \mathcal{F}_{\ell}\right\}$ is a partition of the boundary of $M$ into disjoint collections, then it induces a partition $\left\{\widetilde{\mathcal{F}}_{2}, \ldots \widetilde{\mathcal{F}}_{\ell}\right\}$ of the boundary of $2{ }_{\mathcal{F}_{1}} M$ with one less element. After a finite number of steps, the iteratively doubled manifold is boundaryless and $M$ may be identified with the image of one of the summands (see Theorem 4.2).
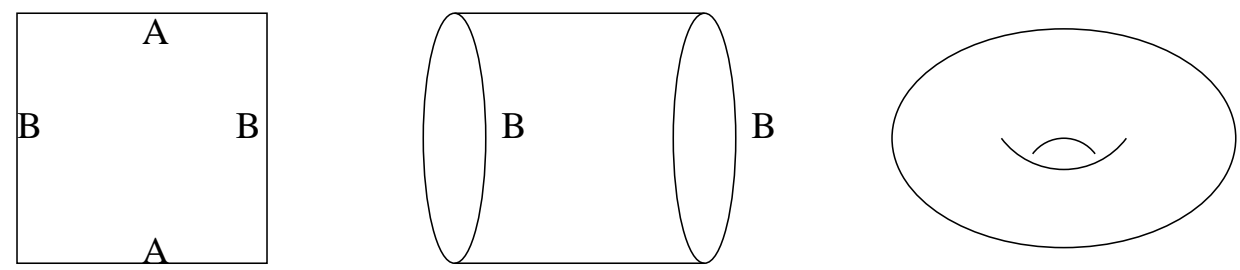

Figure 2. After doubling the boundaries marked $A$ and then doubling the boundaries marked $B$ we end up with a torus.

\section{BLOW UP}

A subset $X \subset M$ of a manifold (with corners) is said to be a p-submanifold if at each point of $X$ there are local (product) coordinates for $M$ such that $X \cap U$, where $U$ is the coordinate neighborhood, is the common zero set of a subset of the coordinates. An interior p-submanifold is a p-submanifold no component of which is contained in the boundary of $M$. 

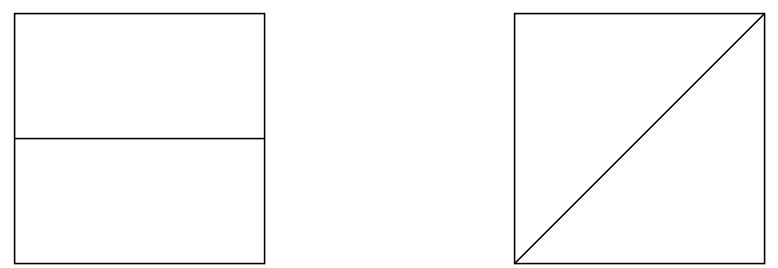

Figure 3. A horizontal line is an interior p-submanifold of the square. The diagonal in a product of manifolds with boundary is not a p-submanifold.

A p-submanifold of a manifold is itself a manifold with corners, and the collar neighborhood theorem holds in this context. Thus the normal bundle to $X$ in $M$ has (for a boundary p-submanifold) a well-defined inward-pointing subset, forming a submanifold with corners $N^{+} X \subset N X$ (defined by the non-negativity of all $d \rho_{H}$ which vanish on the submanifold near the point) and, as in the boundaryless case, the exponential map, but here for a product-type metric, gives a diffeomorphism of a neighborhood of the zero section with a neighborhood of $X$ :

$$
T: N^{+} X \supset U^{\prime} \longrightarrow U \subset M .
$$

The radial vector field on $N^{+} X$ induces a vector field $R$ near $X$ which is tangent to all boundary faces.

Proposition 2.1. If $X$ is a closed p-submanifold in a compact manifold then the boundary product structure in Proposition 1.2. for any choice of boundary defining functions, can be chosen so that $V_{H}$ is tangent to $X$ unless $X$ is contained in $H$.

Proof. The condition that the $V_{H}$ be tangent to $X$ can be carried along in the inductive proof in Proposition 1.2, starting from the smallest boundary face which meets $X$.

If $X \subset M$ is a closed p-submanifold then the radial blow-up of $M$ along $X$ is a well-defined manifold with corners $[M ; X]$ obtained from $M$ by replacing $X$ by the inward-pointing part of its spherical normal bundle. It comes equipped with the blow-down map

$$
[M ; X]=S^{+} X \sqcup(M \backslash X), \beta:[M ; X] \longrightarrow M .
$$

The preimage of $X, S^{+} X$, is the 'front face' of the blow up, denoted $\mathrm{ff}([M ; X])$. The natural smooth structure on $[M ; X]$, with respect to which $\beta$ is smooth, is characterized by the additional condition that a radial vector field $R$ for $X$, as described above, lifts under $\beta$ (i.e. is $\beta$-related) to $\rho_{\mathrm{ff}} X_{\mathrm{ff}}$ for a defining function $\rho_{\mathrm{ff}}$ and normal vector field $X_{\mathrm{ff}}$ for the new boundary introduced by the blow up.

Except in the trivial cases that $X=M$ or $X \in \mathcal{M}_{1}(M)$ the front face is a 'new' boundary hypersurface of $[M ; X]$ and the preimages of the boundary hypersurfaces of $M$ are unions of the other boundary hypersurfaces of $[M ; X]$; namely the lift of $H$ is naturally $[H ; X \cap H]$. So, in the non-trivial cases and unless $X$ separates some boundary hypersurface into two components, there is a natural identification

$$
\mathcal{M}_{1}([M ; X])=\mathcal{M}_{1}(M) \sqcup\{\mathrm{ff}([M ; X])\}
$$

which corresponds to each boundary hypersurface of $M$ having a unique 'lift' to $[M ; X]$, as the boundary hypersurface which is the closure of the preimage of its 

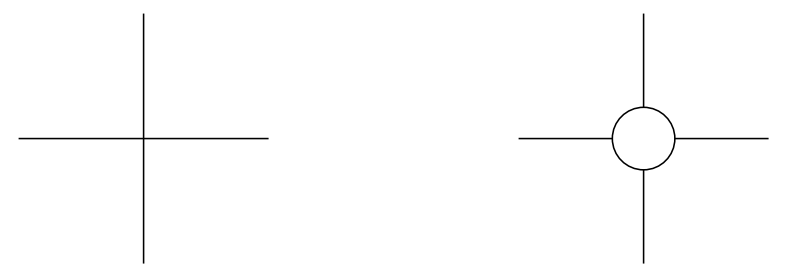

FiguRE 4. Blowing up the origin in $\mathbb{R}^{2}$ results in the manifold with boundary $\left[\mathbb{R}^{2} ;\{0\}\right]=\mathbb{S}^{1} \times \mathbb{R}^{+}$. Polar coordinates around the origin in $\mathbb{R}^{2}$ yield local coordinates near the front face in $\left[\mathbb{R}^{2} ;\{0\}\right]$.

complement with respect to $X$. In local coordinates, blowing-up $X$ corresponds to introducing polar coordinates around $X$ in $M$.

Lemma 2.2. If $X$ is a closed interior p-submanifold and $M$ is equipped with a boundary product structure in the sense of Proposition 1.2 the normal vector fields of which are tangent to $X$ then the radial vector field for $X$ induced by the exponential map of an associated product-type metric commutes with $V_{H}$ near any $H \in \mathcal{M}_{1}(M)$ which intersects $X$ and on lifting to $[M ; X], R=\rho_{\mathrm{ff}} X_{\mathrm{ff}}$ where $\rho_{\mathrm{ff}}$ and $X_{\mathrm{ff}}$, together with the lifts of the $\rho_{H}$ and $V_{H}$ give a boundary product structure on $[M ; X]$.

Proof. After blow up of $X$ the radial vector field lifts to be of the form $a \rho_{\mathrm{ff}} V_{\mathrm{ff}}$ for any normal vector field and defining function for the front face, with $a>0$. The other product data lifts to product data for all the non-front faces of $[M ; X]$ and this lifted data satisfies $\left[R, V_{H}\right]=0$ near ff. Thus it is only necessary to show, using an inductive argument as above, that one can choose $\rho_{\mathrm{ff}}$ to satisfy $V_{H} \rho_{\mathrm{ff}}=0$ and $R \rho_{\mathrm{ff}}=\rho_{\mathrm{ff}}$ in appropriate sets to conclude that $R=\rho_{\mathrm{ff}} V_{\mathrm{ff}}$ as desired.

\section{Resolution structures}

A fibration is a surjective smooth map $\Phi: H \longrightarrow Y$ between manifolds with the property that for each component of $Y$ there is a manifold $Z$ such that each point $p$ in that component has a neighborhood $U$ for which there is a diffeomorphism giving a commutative diagram with the projection onto $U$ :

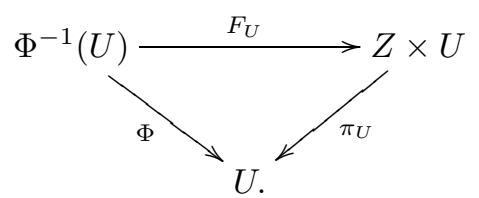

The pair $\left(U, F_{U}\right)$ is a local trivialization of $\Phi$. Set $\operatorname{codim}(\phi)=\operatorname{dim} Z$, which will be assumed to be the same for all components of $Y$. The image of a boundary face under a fibration must always be a boundary face (including the possibility of a component of $Y$ ).

Lemma 3.1. Suppose $\Phi: H \longrightarrow Y$ is a fibration with typical fiber $Z$.

i) If $S \subseteq H$ is a closed p-submanifold transverse to the fibers of $\Phi$, then the composition of $\Phi$ with the blow-down map $\beta:[H ; S] \longrightarrow H$ is a fibration.

ii) If $T \subseteq Y$ is a closed interior p-submanifold, then $\Phi$ lifts from $H \backslash \Phi^{-1}(T)$ to a fibration $\beta^{\#} \Phi:\left[H ; \Phi^{-1}(T)\right] \longrightarrow[Y ; T]$. 
Remark 3.2. In the situation of ii), one may consider instead the pull-back fibration

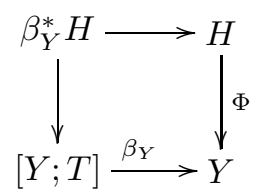

where $\beta_{Y}^{*} H=\left\{(\zeta, \xi) \in H \times[Y ; T]: \Phi(\zeta)=\beta_{Y}(\xi)\right\}$. The natural map $\left[H ; \Phi^{-1}(T)\right] \ni$ $\alpha \mapsto\left(\beta_{H}(\alpha), \widetilde{\Phi}(\alpha)\right) \in \beta_{Y}^{*} H$ is a diffeomorphism, showing that these fibrations coincide.

Proof. i) Transversality ensures that $\Phi(S)=Y$ and so $\left.\Phi\right|_{S}$ is itself a fibration, say with typical fiber $Z_{S}$. If $\left(U, F_{U}\right)$ is a local trivialization of $\Phi$ then since

$$
\left[U \times Z ; U \times Z_{S}\right]=U \times\left[Z ; Z_{S}\right],
$$

the diffeomorphism $F_{U}$ induces a diagram

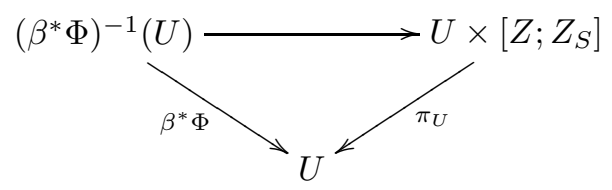

which shows that $\beta^{*} \Phi:[H ; S] \longrightarrow H \longrightarrow Y$ is a fibration.

ii) Let $\left(U, F_{U}\right)$ be a local trivialization of $\Phi$ and $T_{U}=T \cap U$. The diffeomorphism $F_{U}$ identifies $\Phi^{-1}(U)$ with $Z \times U$ and $\Phi^{-1}\left(T_{U}\right)$ with $Z \times T_{U}$ and so lifts to a diffeomorphism $\widetilde{F}_{U}$ of $\left(\beta^{\#} \Phi\right)^{-1}\left(\left[U ; T_{U}\right]\right)$ with $Z \times\left[U ; T_{U}\right]=\left[Z \times U ; Z \times T_{U}\right]$. Thus $\left(\left[U ; T_{U}\right], \widetilde{F}_{U}\right)$ is a local trivialization for $\beta^{\#} \Phi$,

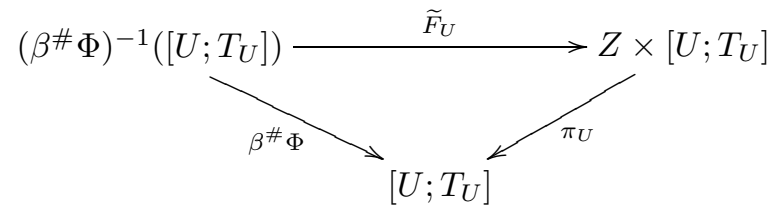

which shows that $\beta^{\#} \Phi:\left[H ; \Phi^{-1}(T)\right] \longrightarrow[Y ; T]$ is a fibration.

The restriction of the blow-down map to the boundary hypersurface introduced by the blow up of a p-submanifold is a fibration, just the bundle projection for the (inward-pointing part of) the normal sphere bundle. In general repeated blow up will destroy the fibration property of this map. However in the resolution of a $G$-action the fibration condition persists. We put this into a slightly abstract setting as follows.

Definition 3.3. A resolution structure on a manifold $M$ is a partition of $\mathcal{M}_{1}(M)$ into collective boundary hypersurfaces, each with a fibration, $\phi_{H}: H \longrightarrow Y_{H}$ with the consistency properties that if $H_{i} \in \mathcal{M}_{1}(M), i=1,2$, and $H_{1} \cap H_{2} \neq \emptyset$ then 
$\operatorname{codim}\left(\phi_{H_{1}}\right) \neq \operatorname{codim}\left(\phi_{H_{2}}\right)$ and

$$
\begin{gathered}
\operatorname{codim}\left(\phi_{H_{1}}\right)<\operatorname{codim}\left(\phi_{H_{2}}\right) \Longrightarrow \\
\phi_{H_{1}}\left(H_{1} \cap H_{2}\right) \in \mathcal{M}_{1}\left(Y_{H_{1}}\right), \phi_{H_{2}}\left(H_{1} \cap H_{2}\right)=Y_{H_{2}} \text { and } \exists \text { a fibration } \\
\phi_{H_{1} H_{2}}: \phi_{H_{1}}\left(H_{1} \cap H_{2}\right) \longrightarrow Y_{H_{2}} \text { giving a commutative diagram: }
\end{gathered}
$$

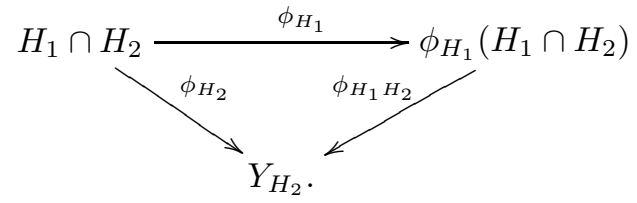

Lemma 3.4. A resolution structure induces resolution structures on each of the manifolds $Y_{H}$.

Proof. Each boundary hypersurface $F$ of $Y_{H}$ is necessarily the image under $\phi_{H}$ of a unique boundary hypersurface of $H$, therefore consisting of a component of some intersection $H \cap K$ for $K \in \mathcal{M}_{1}(M)$. The condition (3.2) ensures that $\operatorname{codim}\left(\phi_{H}\right)<$ $\operatorname{codim}\left(\phi_{K}\right)$ and gives the fibration $\phi_{H K}: F \longrightarrow Y_{K}$. Thus for $Y_{H}$ the bases of the fibrations of its boundary hypersurfaces are all the $Y_{K}$ 's with the property that $H \cap K \neq \emptyset$ and $\operatorname{codim}\left(\phi_{H}\right)<\operatorname{codim}\left(\phi_{K}\right)$ with the fibrations being the appropriate maps $\phi_{*}$ from (3.2).

Similarly the compatibility maps for the boundary fibration of $Y_{H}$ follow by the analysis of the intersection of three boundary hypersurfaces $H, K$ and $J$ where $\operatorname{codim}\left(\phi_{H}\right)<\operatorname{codim}\left(\phi_{K}\right)<\operatorname{codim}\left(\phi_{J}\right)$. Any two intersecting boundary hypersurfaces of $Y_{H}$ must arise in this way, as $\phi_{H}(H \cap K)$ and $\phi_{H}(H \cap J)$ and the compatibility map for them is $\phi_{J K}$.

If $M$ carries a resolution structure then Lemma 3.1 shows that appropriately placed submanifolds can be blown up and the resolution structure can be lifted. Specifically we say that a manifold $T$ is transverse to the resolution structure if either:

i) $T$ is an interior p-submanifold of $M$, with $\operatorname{dim} T<\operatorname{dim} M$, that is transverse to the fibers of $\phi_{H}$ for all $H \in \mathcal{M}_{1}(M)$, or

ii) $T$ is an interior p-submanifold of $Y_{L}$, for some $L \in \mathcal{M}_{1}(M)$, with $\operatorname{dim} T<$ $\operatorname{dim} Y_{L}$, that is transverse to the fibers of $\phi_{N}$ for all $N \in \mathcal{M}_{1}\left(Y_{L}\right)$.

Let $\widetilde{T} \subseteq M$ be equal to $T$ in the first case and $\phi_{L}^{-1}(T)$ in the second, then we have the following result.

Proposition 3.5. If $M$ carries a resolution structure and $T$ is a manifold transverse to it, then $[M ; \widetilde{T}]$ carries a resolution structure. In case ii) above, where $T \subseteq Y_{L}$, the resolution structure on $\left[M ; \phi_{L}^{-1}(T)\right]$ is obtained by blowing-up the lift of $T$ to every $Y_{K}$ that fibers over $Y_{L}$. In both cases, at each boundary face of the new resolution structure the boundary fibration is either the pull-back of the previous one along the blow-down map or the blow-down map itself.

Recall that submanifolds which do not intersect are included in the notion of transversal intersection.

Proof. Consider the two cases in the definition of transverse submanifold separately. (For clarity, we assume throughout the proof that the collective boundary hypersurfaces in Definition 3.3 consist of a single boundary hypersurface.) 
Case i). Let $\beta_{T}:[M ; T] \longrightarrow M$ be the blow-down map. A boundary face of $[M ; T]$ is either the lift of a boundary face $H \in \mathcal{M}_{1}(M)$, in which case $\beta_{T}^{*} \phi_{H}$ is a fibration by Lemma 3.1 i), or it is the front face of the blow-up, in which case it carries the fibration $\left.\beta_{T}\right|_{\mathrm{ff}}$. Thus we only need to check the compatibility conditions.

The compatibility maps for the fibrations of the hypersurfaces of $M$ clearly lift to give compatibility maps for the lifts. Thus it is only necessary to check compatibility between the fibrations on these lifted boundary hypersurfaces of $[M ; T]$ and that of the front face. So, let $H$ be a hypersurface of $M$ that intersects $T$. In terms of the notation above, the codimension of $\beta_{T}^{*} \phi_{H}$ is the equal to $\operatorname{dim} Z_{H}$ while the codimension of $\phi_{\mathrm{ff}}$ is equal to $\operatorname{dim} Z_{H}-\operatorname{dim} Z_{H \cap T}$. The diagram (3.2) in this case is

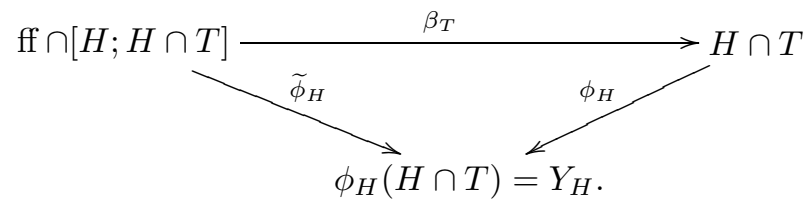

and so the requirements of Definition 3.3 are met.

Case ii). First note that the inverse image of a p-submanifold under a fibration is again a p-submanifold since this is a local property and locally a fibration is a projection. We denote by $\beta_{T}:\left[M ; \phi_{L}^{-1}(T)\right] \longrightarrow M$ the blow-down map and make use of the notation in (3.2).

From the front face the map

$$
\mathrm{ff}\left(\left[M ; \phi_{L}^{-1}(T)\right]\right) \stackrel{\beta_{T}}{\longrightarrow} \phi_{L}^{-1}(T) \stackrel{\phi_{L}}{\longrightarrow} T
$$

is the composition of fibrations and so is itself a fibration.

Consider the lift of a boundary face $H \in \mathcal{M}_{1}(M)$ to a boundary face of $[M ; \widetilde{T}]$. If $H \cap \phi_{L}^{-1}(T)$ is empty then $\beta_{T}^{*} \phi_{H}$ fibers over $Y_{H}$ and the compatibility conditions are immediate. If $H \cap \phi_{L}^{-1}(T)$ is not empty and $\operatorname{codim}\left(\phi_{L}\right)<\operatorname{codim}\left(\phi_{H}\right)$ then, by Lemma 3.1. $\beta_{T}^{*} \phi_{H}$ fibers over $Y_{H}$ and the arrows in the commutative diagrams

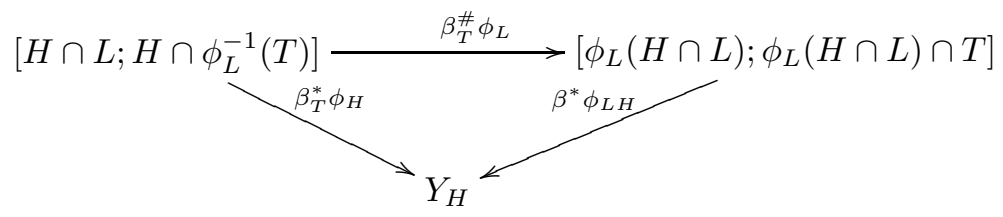

and

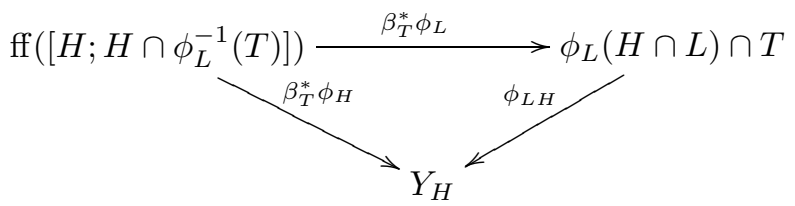

are all fibrations. Here, surjectivity of $\left.\phi_{L H}\right|_{\phi_{L}(H \cap L) \cap T}$ follows from the transversality of $T$ to the fibers of $\phi_{L H}$. Since the lift of $H$ meets the lift of $L$ in $[H \cap$ $\left.L ; H \cap \phi_{L}^{-1}(T)\right]$ and meets the front face of $\left[M ; \phi_{L}^{-1}(T)\right]$ in $\mathrm{ff}\left(\left[H ; \phi_{L}^{-1}(T) \cap H\right]\right)$, these diagrams also establish the compatibility conditions for the lift of $H$.

Next if $H \cap \phi_{L}^{-1}(T)$ is not empty and $\operatorname{codim}\left(\phi_{L}\right)>\operatorname{codim}\left(\phi_{H}\right)$, then Lemma 3.1 guarantees that the map $\beta_{T}^{\#} \phi_{H}$ is a fibration from the lift of $H$ to $\left[Y_{H} ; \phi_{H L}^{-1}(T)\right]$ 
and that the arrows in the commutative diagrams

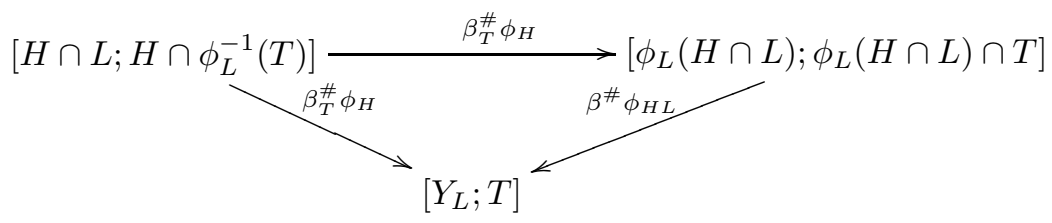

and

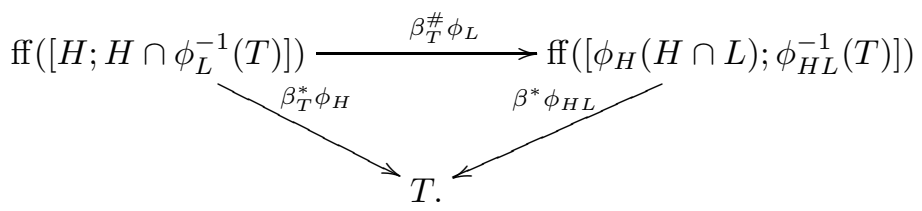

are all fibrations.

Finally consider the lift of $L$. The map $\beta^{\#} \phi_{L}:\left[L ; \phi_{L}^{-1}(T)\right] \longrightarrow\left[Y_{L} ; T\right]$ is a fibration by Lemma 3.1 and the discussion above shows that it is compatible with the lift of $H$ for any $H \in \mathcal{M}_{1}(M)$. The final compatibility between the lift of $L$ and the front face is established by the commutative diagram

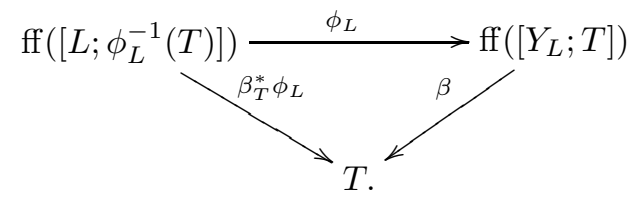

Definition 3.6. If $M$ carries a resolution structure as in Definition 3.3 then a boundary product structure is said to be compatible with the resolution structure if for each pair of intersecting boundary faces $H_{1}$ and $H_{2}$ with $\operatorname{codim}\left(\phi_{H_{1}}\right)<\operatorname{codim}\left(\phi_{H_{2}}\right)$

$$
\begin{gathered}
\left.\rho_{H_{2}}\right|_{H_{1}} \in \phi_{H_{1}}^{*} \mathcal{C}^{\infty}\left(Y_{H_{1}}\right) \text { near } H_{2}, \\
\left.V_{H_{2}}\right|_{H_{1}} \text { is } \phi_{H_{1}} \text {-related to a vector field on } Y_{H_{1}} \text { near } H_{2} \text { and } \\
\left.V_{H_{1}}\right|_{H_{2}} \text { is tangent to the fibers of } \phi_{H_{2}} .
\end{gathered}
$$

Proposition 3.7. For any resolution structure on a compact manifold, $M$, there is a compatible boundary product structure.

Proof. We follow the proof on Proposition 1.2. In particular, we will use the notion of consistent boundary data on a collection of boundary hypersurfaces.

First, choose boundary defining functions satisfying (3.3). Let $H \in \mathcal{M}_{1}(M)$ and define $\mathcal{H} \subset \mathcal{M}_{1}(M)$ to consist of those boundary hypersurfaces $K \in \mathcal{M}_{1}(M)$ which intersect $H$ and satisfy $\operatorname{codim}\left(\phi_{K}\right)<\operatorname{codim}\left(\phi_{H}\right)$. If $L \in \mathcal{H}$, we may assume inductively that we have chosen $\left.\rho_{H}\right|_{K}$ for all boundary hypersurfaces $K \in \mathcal{H}$ with $\operatorname{codim}\left(\phi_{K}\right)<\operatorname{codim}\left(\phi_{L}\right)$, and then choose an extension to $H \cap L$ as a lift of a boundary defining function for the boundary face $\phi_{L}(H \cap L)$. This allows $\rho_{H}$ to be defined on a neighborhood of $H \cap K$ in $K$ for all $K \in \mathcal{H}$; extending it to a boundary defining function of $H$ in $M$ fulfills the requirements.

Next, suppose normal vector fields consistent with the resolution structure and associated collar neighborhoods have been found for some subset $\mathcal{B} \subseteq \mathcal{M}_{1}(M)$ with the property that $H \in \mathcal{M}_{1}(M) \backslash \mathcal{B}$ and $K \in \mathcal{B}$ implies that $H \cap K=\emptyset$ 
or $\operatorname{codim}\left(\phi_{H}\right)<\operatorname{codim}\left(\phi_{K}\right)$. Let $H \in \mathcal{M}_{1}(M) \backslash \mathcal{B}$ be such that $\phi_{H}$ has maximal codimension among the boundary hypersurfaces of $M$ that are not in $\mathcal{B}$. We show that there is a choice of $V_{H}$ and $U_{H}$ such that (1.3) and the conditions of Definition 3.6 hold for all boundary hypersurfaces in $\mathcal{B} \cup\{H\}$.

As before an inductive argument allows us to find $V_{H}$ in a neighborhood of all intersections $H \cap K$ with $K \in \mathcal{B}$ with the property that $\left.V_{H}\right|_{K}$ is tangent to the fibers of $\phi_{K}$. Then $V_{H}$ can be extended into $U_{K}$ using the vector fields $V_{K}$ by demanding that

$$
\mathcal{L}_{V_{K}} V_{H}=\left[V_{K}, V_{H}\right]=0
$$

thus determining $V_{H}$ locally uniquely in a neighborhood of $H \cap K$ in $M$ for all $K \in \mathcal{B}$.

If $K \in \mathcal{M}_{1}(M) \backslash \mathcal{B}$ intersects $H$, then $Y_{K}$ is itself a manifold with a resolution structure and $\phi_{H}(H \cap K)$ is one of its boundary hypersurfaces. We can choose boundary product data on $Y_{K}$ - since it has smaller dimension than $M$ we may assume that the proposition has been proven for it. Under a fibration there is always a smooth lift of vector fields, a connection, so $V_{H}$ on $\phi_{H}(H \cap K)$ may be lifted to a vector field $V_{H}$ on $H \cap K$. In this way $V_{H}$ may be chosen on the intersection of $H$ with any of its boundary faces. Then $V_{H}$ may be extended into a neighborhood $U_{H}$ of $H$ in $M$ in such a way that $V_{H} \rho_{H}=1$. By construction the commutation relations with all the previously constructed vector fields are satisfied and $V_{H}$ is compatible with the resolution structure at all boundary hypersurfaces in $\mathcal{B} \cup\{H\}$. Thus the inductive step is justified.

Using Proposition 2.1 and Lemma 2.2 we see that resolution structures and boundary product structures are preserved when blowing up appropriately placed p-submanifolds.

Proposition 3.8. If $M$ is a manifold with a resolution structure and $X$ is a manifold transverse to the resolution structure then $[M ; \widetilde{X}]$ has a boundary product structure which is compatible with the resolution structure given by Proposition 3.5 , is such that the normal vector fields to boundary hypersurfaces other than the front face are $\beta$-related to a boundary product structure on $M$ and is such that $\rho_{\mathrm{ff}} V_{\mathrm{ff}}$ is $\beta$-related to a radial vector field for $\tilde{X}$.

\section{Group aCtions}

Let $G$ be a compact Lie group and $M$ a compact manifold (with corners). An action of $G$ on $M$ is a smooth map $A: G \times M \longrightarrow M$ such that $A(\mathrm{Id}, \zeta)=\zeta$ for all $\zeta \in M$ and

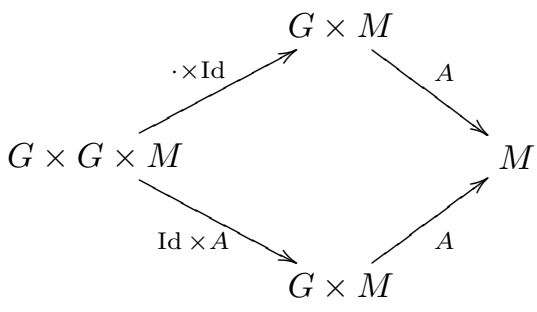

commutes; here - denotes the product in the group. Equivalently this is just the requirement that $A$ induces a group homomorphism from $G$ to the diffeomorphism group of $M$. 
We will usually denote $A(g, \zeta)$ as $g \cdot \zeta$. Since each element $g \in G$ acts as a diffeomorphism on $M$, it induces a permutation of the boundary hypersurfaces of $M$. If $g$ is in the connected component of the identity of $G$, this is the trivial permutation.

Our convention is to assume, as part of the definition, that the action of $G$ is boundary intersection free in the sense of Definition 1.4. That is, the set $\mathcal{M}_{1}(M)$ of boundary hypersurfaces can be partitioned into disjoint sets

$$
\begin{gathered}
\mathcal{M}_{1}(M)=B_{1} \sqcup B_{2} \sqcup \cdots \sqcup B_{l}, H, H^{\prime} \in B_{i} \Longrightarrow H \cap H^{\prime}=\emptyset, \\
\text { and s.t. } g \cdot H \in B_{i} \text { if } H \in B_{i} .
\end{gathered}
$$

The contrary case will be referred to as a $G$-action with boundary intersection it is shown below in Proposition 6.2 that by resolution the boundary intersection can be removed. As justification for our convention, note that the $G$-actions which arise from the resolution of a $G$-action on a manifold without boundary are always boundary intersection free.
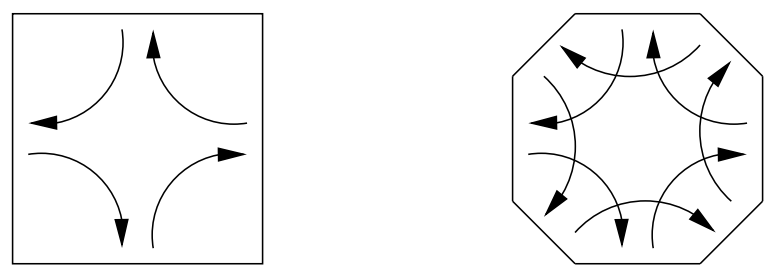

FiguRE 5. The action on the square has boundary intersection, that on the octagon is boundary intersection free.

For a given $G$-action, the isotropy (or stabilizer) subgroup of $G$ at $\zeta \in M$ is

$$
G_{\zeta}=\{g \in G ; g \cdot \zeta=\zeta\}
$$

It is a closed, and hence Lie, subgroup of $G$.

The action of $G$ on $M$ induces a pull-back action on $\mathcal{C}^{\infty}(M)$. The differential of this action at Id $\in G$ induces the action of the Lie algebra $\mathfrak{g}$ on $\mathcal{C}^{\infty}(M)$ where $V \in \mathfrak{g}$ is represented by a vector field $\alpha(V) \in \mathcal{V}_{\mathrm{b}}(M)$, the Lie algebra of smooth vector fields on $M$ tangent to all boundary faces, given by

$$
\alpha(V) f(\zeta)=\left.\frac{d}{d t} f\left(e^{-t V} \zeta\right)\right|_{t=0}, \text { for all } f \in \mathcal{C}^{\infty}(M)
$$

Since $[\alpha(V), \alpha(W)]=\alpha([V, W])$, this is a map of Lie algebras, $\alpha: \mathfrak{g} \longrightarrow \mathcal{V}_{\mathrm{b}}(M)$. The differential at $\zeta \in M$ will be denoted

$$
\alpha_{\zeta}: \mathfrak{g} \longrightarrow T_{\zeta} M
$$

The image always lies in $T_{\zeta} F$ where $F \in \mathcal{M}_{k}(M)$ is the smallest boundary face containing $\zeta$.

Proposition 4.1. For any compact group action on a compact manifold, satisfying (4.2), the collective boundary hypersurfaces $B_{i}$ each have a collective defining function $\rho_{i} \in \mathcal{C}^{\infty}(M)$ which is $G$-invariant and there is a corresponding $G$-invariant 
product structure near the boundary consisting of smooth $G$-invariant vector fields $V_{i}$ and neighborhoods $U_{i}$ of $\operatorname{supp}\left(B_{i}\right)=\cup\left\{H \in B_{i}\right\}$ for each $i$ such that

$$
V_{i} \rho_{j}= \begin{cases}1 & \text { in } U_{i} \text { if } i=j \\ 0 & \text { in } U_{i} \cap U_{j} \text { if } i \neq j .\end{cases}
$$

Furthermore there is a $G$-invariant product-type metric on $M$.

Proof. Any collective boundary hypersurface has a common defining function, given by any choice of defining function near each boundary hypersurface in the set extended to be strictly positive elsewhere. If $\rho_{i}^{\prime}$ is such a defining function for $\operatorname{supp}\left(B_{i}\right)$ then so is $g^{*} \rho_{i}$ for each $g \in G$, since by assumption it permutes the elements of $B_{i}$. Averaging over $G$ gives a $G$-invariant defining function. Similarly each of the vector fields $V_{H}$ in (1.3) is only restricted near $H$ so these can be combined to give collective normal vector fields $V_{i}$ which then have the properties in (4.6). Since the commutation conditions are bilinear they cannot be directly arranged by averaging, but the normal vector fields can be constructed, and averaged, successively.

A product-type metric made up (iteratively) from this invariant data near the boundary can similarly be averaged to an invariant product-type metric. In fact the average of any metric for which the boundary faces are all totally geodesic has the same property.

One direct consequence of the existence of an invariant product structure near the boundary is that, as noted above, a smooth group action on a manifold with corners can be extended to a group action on a closed manifold. This allows the consideration of the standard properties of group actions to be extended trivially from the boundaryless case to the case considered here of manifolds with corners.

Theorem 4.2. Suppose $M$ is a compact manifold with corners with a smooth action by a compact Lie group $G$ - so assumed to satisfy (4.2) - then if $M$ is doubled successively, as at the end of $\$ 1$, across the elements of a partition into $l G$-invariant collective boundary hypersurfaces, to a manifold without boundary, $\widehat{M}$, then there is a smooth action of $\mathbb{Z}_{2}^{l} \times G$ on $\widehat{M}$ such that $M$ embeds $G$-equivariantly into $\widehat{M}$ as a fundamental domain for the $\mathbb{Z}_{2}^{l}$-action.

Proof. (See 12, Chapter 1] and 4, §II.1]) A partition of $\mathcal{M}_{1}(M)$ of the stated type does exist, as in (4.2). Proposition 4.1 shows the existence of a $G$-invariant product-type metric, collective boundary defining functions and product decompositions near the boundary hypersurfaces. First consider the union of two copies of $M$, denoted $M^{ \pm}$, with all points in $\operatorname{supp}\left(B_{1}\right)$, i.e. all points in the boundary hypersurfaces in $B_{1}$, identified

$$
M_{1}=\left(M^{+} \sqcup M^{-}\right) / \simeq_{1}, p \simeq_{1} p^{\prime}, p=p^{\prime} \text { in } H \in B_{1} .
$$

Now, the local product decompositions near each element of $B_{1}$ induce a $\mathcal{C}^{\infty}$ structure on $M_{1}$ making it again a manifold with corners. Thus $\rho_{1}$, the collective defining function for $B_{1}$ on $M=M^{+}$can be extended to the smooth function

$$
\rho_{1}^{\prime}= \begin{cases}\rho_{1} & \text { on } M^{+} \\ -\rho_{1} & \text { on } M^{-} .\end{cases}
$$

Similarly the corresponding normal vector field $V_{1}$ extends to be smooth when defined as $-V_{1}$ on $M^{-}$. The action of $G$ on $M$ gives actions on $M^{ \pm}$which are consistent on $\operatorname{supp}\left(B_{1}\right)$ and the product decomposition of the group action shows that 
the combined action on $M_{1}$ is smooth. The boundary hypersurfaces of $M_{1}$ fall into two classes. Those arising from boundary hypersurfaces of $M$ which meet one of the elements of $B_{1}$, these appear as the doubles of the corresponding hypersurfaces from $M$. The boundary hypersurfaces of $M$ which do not meet an element of $B_{1}$ contribute two disjoint boundary hypersurfaces to $M_{1}$. It follows that the decomposition of $\mathcal{M}_{1}(M)$ in (4.2) induces a similar decomposition of $\mathcal{M}_{1}\left(M_{1}\right)$ in which each $B_{i}, i=2, \ldots, l$ contains the preimages of the boundary hypersurfaces of $M$, other than the elements of $B_{1}$, under the natural projection $M_{1} \longrightarrow M$. The $\mathbb{Z}_{2}$ action on $M_{1}$ given by exchanging signs is smooth, by construction, and commutes with the $G$-action.

Thus this procedure can be repeated $l$ times finally giving a manifold without boundary with smooth $G$-action as desired.

\section{INVARIANT TUBES AND COLLARS}

As note above the doubling construction allows the standard properties of group actions on boundaryless manifolds to be transferred to the context of manifolds with corners. In fact the standard proofs may also be extended directly.

If $\zeta \in M$ then the stabilizer $G_{\zeta}$ acts on $T_{\zeta} M$ and on the metric balls, of an invariant product-type metric, in $T_{\zeta} M$. If $\zeta$ is contained in a corner of codimension $k \geq 0$, then the exponential map for the metric identifies the set of the inwardpointing vectors in a small ball in $T_{\zeta} M$ with a $G_{\zeta}$-invariant neighborhood of $\zeta$ in $M$ hence establishes the basic linearization result.

Proposition 5.1 (Bochner). If $\zeta \in M$ is contained in a corner of codimension $k \geq 0$ then there is a $G_{\zeta}$-invariant neighborhood $\mathcal{U}_{\zeta}$ of $\zeta$ in $M$, a linear action $\alpha_{\zeta}$ of $G_{\zeta}$ on $\mathbb{R}^{m, k}$, and a $G_{\zeta}$-equivariant diffeomorphism $\chi_{\zeta}: \mathcal{U}_{\zeta} \longrightarrow B^{+}$to (the inward-pointing part of) a ball $B^{+} \subset \mathbb{R}^{m, k}$.

Corollary 5.2. If $G$ is a compact Lie group acting smoothly on a manifold $M$, then $M^{G}=\{\zeta \in M ; g \cdot \zeta=\zeta$ for all $g \in G\}$ is an interior p-submanifold of $M$.

A slice at $\zeta \in M$ for the smooth action of $G$ is a p-submanifold, $S$, of $M$ through $\zeta$ such that

i) $T_{\zeta} M=\alpha_{\zeta}(\mathfrak{g}) \oplus T_{\zeta} S$,

ii) $T_{\zeta^{\prime}} M=\alpha_{\zeta^{\prime}}(\mathfrak{g})+T_{\zeta^{\prime}} S$ for all $\zeta^{\prime} \in S$,

iii) $S$ is $G_{\zeta}$-invariant,

iv) If $g \in G$ and $\zeta^{\prime} \in S$ are such that $g \cdot \zeta^{\prime} \in S$ then $g \in G_{\zeta}$.

For $\varepsilon \in(0,1)$, set

$$
S_{\varepsilon}=\chi_{\zeta}^{-1}\left(\alpha_{\zeta}(\mathfrak{g})^{\perp} \cap B^{+}(\varepsilon)\right)
$$

where $B^{+}(\epsilon) \subset T_{\zeta} M$ is the set of inward-pointing vectors of length less than $\varepsilon$. Since the vector fields in the image of $\alpha$ are tangent to all of the boundary faces, $S_{\varepsilon}$ is necessarily a p-submanifold of $M$ through $\zeta$. Elements $k \in G_{\zeta}$ satisfy $T_{\zeta} A(k)\left(\alpha_{\zeta}(X)\right)=\alpha_{\zeta}(\operatorname{Ad} k(X))$, so the tangent action of $G_{\zeta}$ preserves $\alpha_{\zeta}(\mathfrak{g})$ and hence $S_{\varepsilon}$ is $G_{\zeta}$-invariant. The Slice Theorem for boundaryless manifolds [7, Theorem 2.3.3], applied to $\widehat{M}$, shows that $S_{\varepsilon}$ is a slice for the $G$-action at $\zeta$ if $\varepsilon$ is small enough.

Similarly, the following result is [7, Theorem 2.4.1] applied to $\widehat{M}$. 
Proposition 5.3 (Tube Theorem). If $G$ acts smoothly on a manifold $M$ and $\zeta \in M$, then there is a representation space $V$ of $G_{\zeta}$ with $G_{\zeta}$-invariant subset $V^{+}$of the form $\mathbb{R}^{\ell, k}$, a $G$-invariant neighborhood $U$ of $\zeta \in M$, a $G_{\zeta}$-invariant neighborhood, $V$, of the origin in $V^{+}$and a $G$-equivariant diffeomorphism

$$
\phi: G \times_{G_{\zeta}} V^{+} \longrightarrow U \text { s.t. } \phi(0)=\zeta .
$$

It is straightforward to check (see [11, Lemma 4.16]) that the $G$-isotropy group of $[(g, v)] \in G \times \times_{G_{\zeta}} V$ is conjugate (in $G$ ) to the $G_{\zeta}$ isotropy group of $v$ in $V$. Thus, if $U$ is a neighborhood of $\zeta$ as in Proposition 5.3 and $\zeta^{\prime} \in U$, then

$$
G_{\zeta^{\prime}} \text { is conjugate to a subgroup of } G_{\zeta} \text {. }
$$

Exponentiation using a product-type $G$-invariant metric allows a neighborhood of a $G$-invariant p-submanifold $X \subseteq M$ to be identified with a neighborhood of the zero section of its normal bundle.

Proposition 5.4 (Collar Theorem). If $G$ acts smoothly on a manifold $M$ and $X \subseteq M$ is a $G$-invariant interior p-submanifold, then there exists a $G$-invariant neighborhood $U$ of $X$ in $M$ and a $G$-invariant diffeomorphism from the normal bundle $N X$ of $X$ to $U$ that identifies the zero section of $N X$ with $X$ and for all sufficiently small $\varepsilon>0$ the submanifolds

$$
\mathcal{S}_{\varepsilon}(X)=\{\zeta \in M ; d(\zeta, X)=\varepsilon\}
$$

are $G$-invariant and the $G$-actions on $S_{\varepsilon}(X)$ and $S_{\varepsilon^{\prime}}(X)$ are intertwined by translation along geodesics normal to $X$.

Proof. As a p-submanifold, $X$ has a tubular neighborhood in $M$, which by exponentiating we can identify with

$$
U_{\varepsilon}=\{\zeta \in M ; d(\zeta, X) \leq \varepsilon\} .
$$

For $\varepsilon$ small enough, each $\zeta \in U_{\varepsilon}$ is connected to $X$ by a unique geodesic of length less than $\varepsilon, \gamma_{\zeta}$. Since the $G$-action is distance preserving and short geodesics are the unique length-minimizing curves between their end-points,

$$
g \cdot \gamma_{\zeta}=\gamma_{g \cdot \zeta}, \text { for every } g \in G, \zeta \in U_{\varepsilon} .
$$

It follows that $G$ preserves $S_{\varepsilon^{\prime}}(X)$ for all $\varepsilon^{\prime}<\varepsilon$ and that translation along geodesics normal to $X$ intertwines the corresponding $G$-actions, as claimed.

If $\Phi: M \longrightarrow Y$ is an equivariant fibration and $G$ acts trivially on $Y$, we can find a $G$-invariant submersion metric. Exponentiating from a fiber of $\Phi, \Phi^{-1}(q)$, gives an equivariant identification with a neighborhood of the form $\Phi^{-1}(q) \times U$, which establishes the following result.

Proposition 5.5. Suppose $G$ acts on the manifolds $M$ and $Y, \Phi: M \longrightarrow Y$ is an equivariant fibration, and the action of $G$ on $Y$ is trivial, then the fibers of $\Phi$ are G-equivariantly diffeomorphic.

\section{Boundary RESOLUTION}

In this section the first steps towards resolution of a group action by radial blow-up are taken. Namely it is shown that on the blow-up of a $G$-invariant closed p-submanifold, $X$, the group action extends smoothly, and hence uniquely, from $M \backslash X$ to $[M ; X]$; the blow-down map is then equivariant. Using this it is then shown that any smooth action, not requiring (4.2), on a manifold with corners 
lifts to a boundary intersection free action, i.e. one which does satisfy (4.2), after blowing-up appropriate boundary faces.

Let $\mathcal{J}(M)$ be the set of isotropy groups for a smooth action of $G$ on $M$.

Proposition 6.1. If $X \subseteq M$ is a $G$-invariant closed p-submanifold for a smooth action by a compact Lie group, $G$, on $M$ then $[M ; X]$ has a unique smooth $G$-action such that the blow-down map $\beta:[M ; X] \longrightarrow M$ is equivariant and

$$
\mathcal{J}([M ; X])=\mathcal{J}(M \backslash X) .
$$

Proof. The blown-up manifold is

$$
[M ; X]=N^{+} X \sqcup(M \backslash X)
$$

with smooth structure consistent with the blow up of the normal bundle to $X$ along its zero section. Thus $[M ; X]$ is diffeomorphic to $M \backslash U_{\varepsilon}$ with $U_{\varepsilon}$ as in (5.2). This diffeomorphism induces a smooth $G$-action on $[M ; X]$ with respect to which the blow-down map is equivariant. The result for isotropy groups, (6.1), follows from (5.1), namely the isotropy groups away from the front face of $[M ; X]$ are certainly identified with those in $M \backslash X$ and the isotropy groups on $\mathrm{ff}([M ; X])$ are identified with those in $S_{\varepsilon}$ for small $\varepsilon>0$.

A general smooth group action will lift to be boundary intersection free on the total boundary blow-up of $M$. This manifold $M_{\mathrm{tb}}$, discussed in [8, §2.6], is obtained from $M$ by blowing-up all of its boundary faces, in order of increasing dimension. Blowing up all of the faces of dimension less than $k$ separates all of the faces of dimension $k$ so these can be blown-up in any order without changing the final space which is therefore well-defined up to canonical diffeomorphism.

In Figure 4 the octagon is obtained from the square by blowing-up the corners and the $\mathbb{Z} / 4$-action lifts from the square to the boundary intersection free action on the octagon.

Proposition 6.2. If $G$ acts smoothly on a manifold $M$, without necessarily satisfying (4.2), the induced action of $G$ on $M_{\mathrm{tb}}$ is boundary intersection free, i.e. does satisfy (4.2).

Proof. Let $\beta: M_{\mathrm{tb}} \longrightarrow M$ be the blow-down map. Any boundary hypersurface $Y$ of $M_{\mathrm{tb}}$ is the lift of a boundary face $F=\beta(Y)$ of $M$. Since each element $G$ acts on $M$ by a diffeomorphism it sends $\beta(Y)$ to a boundary face of $M$ of the same dimension as $F$, say $F^{\prime}=\beta\left(Y^{\prime}\right)$. The induced action on $M_{\mathrm{tb}}$ will send the boundary face $Y$ to $Y^{\prime}$ and, from the definition of $M_{\mathrm{tb}}, Y^{\prime}$ is either equal to $Y$ or disjoint from $Y$. Hence the action of $G$ on $M_{\mathrm{tb}}$ is boundary intersection free.

In fact it is generally possible to resolve an action to be boundary intersection free by blowing up a smaller collection of boundary faces. Namely, consider all the boundary faces which have the property that they are a component of an intersection $H_{1} \cap \cdots \cap H_{N}$ where the $H_{i} \in \mathcal{M}_{1}(M)$ are intertwined by $G$, meaning that for each $1 \leq i<j \leq N$ there is an element $g_{i j} \in G$ such that $g_{i j}\left(H_{j}\right)=H_{i}$. This collection of boundary faces satisfies the chain condition that if $F$ is an element and $F^{\prime} \supset F$ then $F^{\prime}$ is also an element. In fact this collection of boundary faces is divided into transversal subcollections which are closed under intersection and as a result the manifold obtained by blowing them up in order of increasing dimension is well-defined. It is straightforward to check that the lift of the $G$-action to this partially boundary-resolved manifold is boundary intersection free. 


\section{Resolution of $G$-ACtions}

The set, $\mathcal{J}(M)$, of isotropy groups which occur in a smooth $G$-action is necessarily closed under conjugation, since if $G_{\zeta} \in \mathcal{J}$ then $G_{g \zeta}=g G_{\zeta} g^{-1}$. Let $\mathcal{I}=\mathcal{J} / G$ be the set of conjugacy classes of isotropy groups for the action of $G$ on $M$ and for each $I \in \mathcal{I}$ let

$$
M^{I}=\left\{\zeta \in M ; G_{\zeta} \in I\right\},
$$

be the corresponding isotropy type. Proposition 5.3 shows these to be smooth psubmanifolds and they stratify $M$, with a natural partial order

$$
I^{\prime} \preccurlyeq I \text { or } M^{I} \preccurlyeq M^{I^{\prime}} \text { if } K \in I \text { is conjugate to a subgroup of an element of } I^{\prime} \text {. }
$$

Thus minimal elements with respect to $\preccurlyeq$ are the ones with the largest isotropy groups. We also set

$$
M_{I}=\operatorname{cl}\left(M^{I}\right) \subset \bigcup_{I^{\prime} \preccurlyeq I} M^{I^{\prime}}
$$

Proposition 7.1. The isotropy types $M^{I} \subset M$ for a smooth action by a compact group $G$ form a finite collection of p-submanifolds each with finitely many components.

Proof. In [7, Proposition 2.7.1], this result is shown for boundaryless manifolds. By passing from $M$ to $\widehat{M}$ as in Theorem 4.2 , the same is true for manifolds with corners with the local product condition implying that $M^{I}$ is a p-submanifold following from Proposition 5.2 .

Definition 7.2. A resolution of a smooth $G$-action on a compact manifold $M$ (with corners) is a manifold, $Y$, obtained by the successive blow up of closed $G$-invariant p-submanifolds of $M$ to which the $G$-action lifts to have a unique isotropy type.

Proposition 6.1 shows that there is a unique lifted $G$-action such that the iterated blow-down map is $G$-equivariant.

Such a resolution is certainly not unique - as in the preceding section, in the case of manifolds with corners, it is always possible to blow up a boundary face in this way, but this is never required for the resolution of an action satisfying (4.2). We show below that there is a canonical resolution obtained by successively blowing up minimal isotropy types. To do this we note that the blow-ups carry additional structure.

Definition 7.3. An equivariant resolution structure for a $G$ action on a manifold $Y$ is a resolution structure, in the sense of Definition 3.3, with $G$-equivariant fibrations to bases each with unique isotropy type and such that in addition none of the isotropy types in any base is present in the total space. A full resolution for a $G$-action on a manifold, $M$, is a resolution in the sense of Definition 7.2 where $Y$ carries such an equivariant resolution structure.

Proposition 7.4. Let $M$ be a smooth manifold with a smooth boundary intersection free action by a compact Lie group $G$ and an equivariant resolution structure, then any minimal isotropy type $X=M^{I}$ is a closed interior $p$-submanifold and if it is transversal to the fibers of all the boundary fibrations then $[M ; X]$ has an induced equivariant resolution structure. 
Proof. As for a boundaryless manifold the minimal isotropy type is closed in $M$ since its closure can only contain points with larger isotropy group. It is an interior p-submanifold by Proposition 7.1 thus the blow up $[M ; X]$ is well-defined. The $G$-action lifts smoothly to $[M ; X]$ by Proposition 6.1 and the defining isotropy type $I$ is not present in the resolved action. The assumed transversality allows Proposition 3.5 i) to be applied to conclude that the resolution structure lifts to $[M ; X]$ and so gives an equivariant resolution structure.

Theorem 7.5. A compact manifold (with corners), $M$, with a smooth, boundary intersection free, action by a compact Lie group, $G$, has a canonical full resolution, $Y(M)$, obtained by iterative blow-up of minimal isotropy types.

Proof. In view of Proposition 7.4 it only remains to show, iteratively, that at each stage of the resolution any minimal isotropy type is transversal to the fibers of the earlier blow ups.

At the first step the transversality condition is trivial, since there is no boundary, and so the first blow-up can be carried out and leads to an equivariant resolution structure. Thus we can assume, inductively, that the equivariant resolution structure exists at some level and then we simply need to check that any minimal isotropy type for the lifted action is transversal to the fibers of each of the fibrations. Transversality is a local condition and at a point of boundary codimension greater than one the compatibility condition for a resolution structure ensures that the fibration of one of the boundary hypersurfaces through that point has smallest leaves and it is necessarily the 'most recent' blow up. Thus we need only consider the case of a point of intersection of the minimal isotropy type and the front face produced by the blow up of an earlier minimal isotropy type in which there are (locally) no intermediate blow ups. Working locally, in the manifold before the earlier of the two blow ups, we simply have a manifold with a $G$-action and two intersecting isotropy types, one of which is locally minimal.

Now, by Proposition 5.3, if $\zeta$ is such a point of intersection, with isotropy group $H$, it has a neighborhood, $U$, with a $G$-equivariant diffeomorphism to $L=G \times_{H} V^{+}$ with $V^{+}$the inward-pointing unit ball in a representation space $V$ for $H$. The points in $V$ with isotropy group $H$ form a linear subspace and $H$ acts on the quotient. Thus the action is locally equivariantly diffeomorphic to $G \times_{H} W^{+} \times B$ where the action is trivial on $B$ and $W^{+} \subset W$ is a ball around the origin in a vector space $W$ with linear $H$-action such that $W^{H}=\{0\}$. Thus any isotropy type meeting $M^{H}$ at $\zeta$ is represented as a twisted product by $G \times{ }_{H}\left(V^{+}\right)^{I} \times B$ where $I$ is an isotropy class in $H$. In particular such a neighboring isotropy type is a bundle over the minimal isotropy type and meets the fibers of a normal sphere bundle of small radius transversally. Thus, on blow-up it meets the fibers of the front face, which are these spheres, transversally.

Thus in fact the successive blow-ups are always transversal to the fibers of the early ones and hence the successive partial resolution structures lift and finally give a full resolution.

The uniqueness of this full resolution follows from the fact that at each stage the alternative is to blow up one of a finite set of minimal isotropy types. Since these are disjoint the order at this stage does not matter and hence, inductively, any such order produces a canonically diffeomorphic full resolution. 
Remark 7.6. As mentioned in the introduction, there is a one-to-one correspondence between the isotropy types of the $G$-action on $M$ and the $G$-invariant collective boundary hypersurfaces in the resolution structure of $Y(M)$. The base of the boundary fibration corresponding to the isotropy type $M^{[K]}$ is the canonical resolution of $M_{[K]}$, the closure of $M^{[K]}$ in $M$, i.e., $Y_{[K]}(M)=Y\left(M_{[K]}\right)$. If $M$ is connected, the isotropy type of $Y(M)$ is the unique open, or principal, isotropy type of $M$, so $M$ and $Y(M)$ can be thought of as different compactifications of the same open set.

Consider the action of $\mathbb{S}^{1}$ on $\mathbb{S}^{2}$ by rotation around the $z$-axis. There are two isotropy types: one consisting of the 'north pole' and 'south pole', $\{N, S\}$, has isotropy group $\mathbb{S}^{1}$, while the complement has isotropy group $\{\operatorname{Id}\}$. The resolution is obtained by blowing-up the former isotropy type and keeping the blow-down maps as the boundary fibration,

$$
Y\left(\mathbb{S}^{2}\right)=\left[\mathbb{S}^{2} ;\{N, S\}\right]
$$
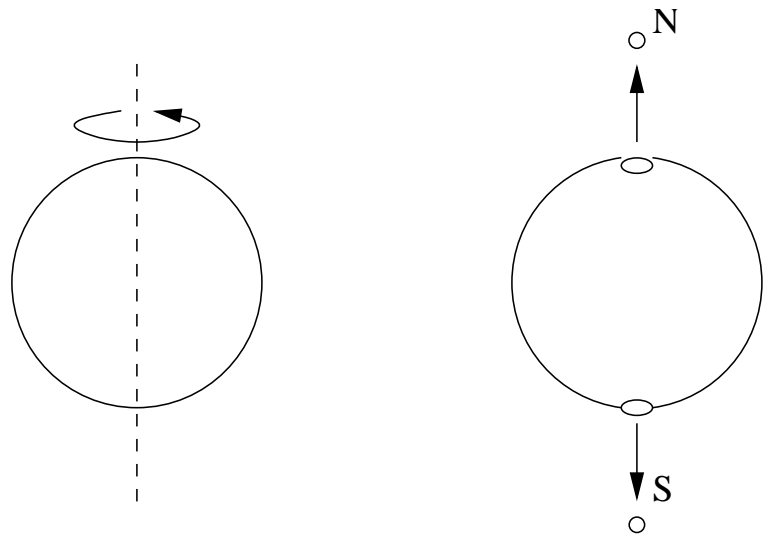

FiguRE 6 . The resolution of the $\mathbb{S}^{1}$ action on $\mathbb{S}^{2}$.

In non-trivial cases, the resolution of a product of $G$-actions is not equal to the product of the resolutions. For instance, consider the $\mathbb{Z}_{2}$-action on $[-1,1]$ given by reflecting across the origin and the product action of $\mathbb{Z}_{2} \times \mathbb{Z}_{2}$ on $[-1,1] \times[-1,1]$. The resolution of $[-1,1]$ is

$$
Y([-1,1])=[[-1,1] ;\{0\}]=[-1,0] \sqcup[0,1]
$$

while the resolution of $\mathbb{R}^{2}$ is

$$
Y\left(\mathbb{R}^{2}\right)=\left[[-1,1]^{2} ;\{(0,0)\} ;[-1,1] \times\{0\} ;\{0\} \times[-1,1]\right]
$$

which in particular is not equal to $Y([-1,1])^{2}$.

\section{Resolution OF ORBit SPACES}

Having constructed a resolution of the group action, we now view the consequences for the orbit space. For boundaryless manifolds with a unique isotropy type Borel showed that the orbit space is a smooth manifold, and the natural projection onto it is a smooth fibration, though in the non-free case not a principal bundle. It is straight-forward to extend this to manifolds with corners. 

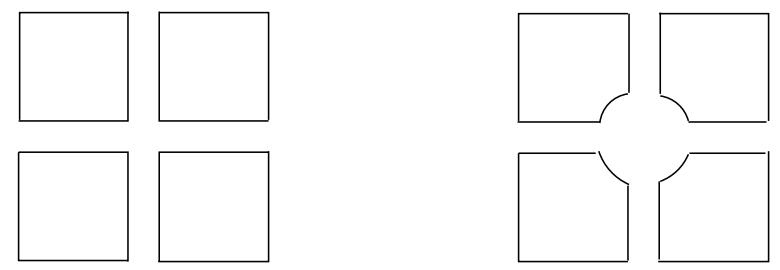

Figure 7. With these actions, $Y([-1,1])^{2}$ is the disjoint union of four quadrants and $Y\left([-1,1]^{2}\right)$ is the disjoint union of four quadrants with a corner blown-up.

Proposition 8.1 (Borel). Let $M$ be a manifold with a (boundary intersection free) $G$-action with a unique isotropy type, if $N(K)$ is the normalizer of an isotropy group $K$ then $M$ is $G$-equivariantly diffeomorphic to $G \times_{N(K)} M^{K}$ and the inclusion $M^{K} \hookrightarrow M$ induces a diffeomorphism

$$
(N(K) / K) \backslash M^{K}=N(K) \backslash M^{K} \cong G \backslash M .
$$

Proof. We follow the proof of [7, Theorem 2.6.7] in the boundaryless case. It is shown in Corollary 5.2 that for a fixed isotropy group $M^{K}$ is a smooth interior psubmanifold. The normalizer $N(K)$ acts on $M^{K}$ with isotropy group $K$ so the quotient group $W(K)=N(K) / K$ acts freely on $M^{K}$. Thus the quotient $W(K) \backslash M^{K}$ is smooth. The diagonal action of $N(K)$ on the product

$$
N(K) \times\left(G \times M^{K}\right) \ni(n,(g, m)) \longmapsto\left(g n^{-1}, n m\right) \in G \times M^{K}
$$

is free, so the quotient $G \times_{N(K)} M^{K}$ is also smooth. Moreover the action of $G$ on $M$ factors through the quotient, $g m=g n^{-1} \cdot n m$, so defines the desired smooth map

$$
G \times_{N(K)} M^{K} \longrightarrow M
$$

This is clearly $G$-equivariant for the left action of $G$ on $G \times_{N(K)} M^{K}$ and is the identity on the image of $\{\operatorname{Id}\} \times M^{K}$ to $M^{K}$. The Slice Theorem shows that the inverse map, $m \longmapsto\left[\left(g, m^{\prime}\right)\right]$ if $m^{\prime} \in M^{K}$ and $g m^{\prime}=m$ is also smooth, so (8.2) is a $G$-equivariant diffeomorphism.

The quotient $G \backslash\left(G \times_{N(K)} M^{K}\right)=N(K) \backslash M^{K}$ is smooth and the smooth structure induced on $G \backslash M$ is independent of the choice of $K$.

Thus if $Y(M)$ is the canonical resolution of the $G$-action on $M$, the orbit space

$$
Z(M)=G \backslash Y(M)
$$

is a smooth manifold with corners, as is the orbit space, $Z_{I}$, of each $Y_{I}$. Moreover, the boundary hypersurfaces of $Z(M)$ may be identified with the equivalence classes under the action of $G$ of the boundary hypersurfaces of $Y(M)$ and the boundary fibrations of $Y(M)$, being $G$-equivariant, descend to give a resolution structure on $Z(M)$.

\section{EquiVARIANT MAPS AND RESOLUTION}

Given two manifolds with $G$-actions and an equivariant map between them, there need not be a corresponding map between their canonical resolutions. Any map can be factored into the composition of an embedding followed by a fibration, and 
in this section we describe the relation between these maps and resolution. In particular we will discuss the resolution of a space with respect to an equivariant fibration.

The behavior of resolution with respect to embeddings is particularly simple.

Theorem 9.1. Let $X$ and $M$ be manifolds with $G$-actions, and let $i: X \hookrightarrow M$ be an equivariant embedding of $X$ as a p-submanifold of $M$. Let $[K]$ be the open isotropy type of $X$, so that $i(X) \subseteq M_{[K]}$, and let

$$
Y_{[K]}(M) \stackrel{\beta_{[K]}}{\longrightarrow} M_{[K]}
$$

be the resolution of $M_{[K]}$. Then

$$
Y(X)=\overline{\beta_{[K]}^{-1}\left(X_{[K]}\right)},
$$

where the closure is taken in $Y_{[K]}(M)$, and so we have a commutative diagram

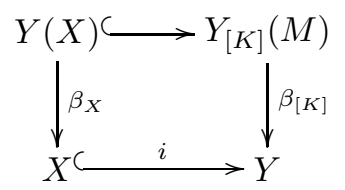

Proof. It suffices to note that, if $S, X \subset M$ are closed p-submanifolds with a common local product description, so $S \cap X \subset X$ is a p-submanifold and if $\gamma$ : $[M ; S] \longrightarrow M$ is the blow-down map, then

$$
\overline{\gamma^{-1}(X \backslash S)}=[X ; X \cap S] \text {. }
$$

Thus, since $X_{[L]}=M_{[L]} \cap X$ for every subgroup $L$ of $G$, resolving $M_{[K]}$ simultaneously resolves $X=X^{[K]}$.

We next consider the resolution of the total space of a fibration, first without group actions.

Suppose that $X$ and $M$ carry resolution structures and $f: X \longrightarrow M$ is a fibration with the property that, for each $H \in \mathcal{M}_{1}(X)$ such that $f(H) \in \mathcal{M}_{1}(X), f$ maps the fibers of $\phi_{H}$ to the fibers of $\phi_{f(H)}$. Thus $f$ induces a fibration $\bar{f}_{H}: Y_{H} \longrightarrow Y_{f(H)}$ covered by $\left.f\right|_{H}$. In this case we say that $f$ is a resolution fibration. Note that for $H \in \mathcal{M}_{1}(X), f(H)$ is either a boundary hypersurface of $M$ or a component of $M$.

Lemma 9.2. Suppose $X$ and $M$ carry resolution structures and $f: X \longrightarrow M$ is a resolution fibration.

i) If $S \subseteq X$ is a closed p-submanifold transverse to the fibers of $f$ and to the fibers of $\phi_{H}$ for each $H \in \mathcal{M}_{1}(X)$, then the composition of $f$ with the blow-down map $[X ; S] \longrightarrow X \longrightarrow M$ is a resolution fibration.

ii) If $T \subseteq M$ is a closed interior p-submanifold transverse to the fibers of each $\phi_{K}$ for $K \in \mathcal{M}_{1}(M)$, then $f$ lifts from $X \backslash f^{-1}(T)$ to a resolution fibration $\left[X ; f^{-1}(T)\right] \longrightarrow[M ; T]$.

iii) If $L \subseteq Y_{H}$ is an interior p-submanifold (with $\operatorname{dim} L<\operatorname{dim} Y_{H}$ ) for some $H \in \mathcal{M}_{1}(X)$ and $\phi_{H}^{-1}(L)$ is transverse to the fibers of $f$ then the composition, $\left[X ; \phi_{H}^{-1}(L)\right] \longrightarrow X \longrightarrow M$, of $f$ with the blow-down map is a resolution fibration. 
Proof. For i), it only remains to establish that $[X ; S] \longrightarrow X \longrightarrow M$ is a resolution fibration. If $H \in \mathcal{M}_{1}(X)$ intersects $S$ then $\phi_{H}$ restricts to $S \cap H$ to a fibration $Z_{S} \longrightarrow S \cap H \longrightarrow Y_{H}$ and on passing from $X$ to $[X ; S]$ the boundary fibration $Z \longrightarrow H \longrightarrow Y_{H}$ is replaced with $\left[Z ; Z_{S}\right] \longrightarrow[H ; H \cap S] \longrightarrow Y$. Thus the blow-down map sends the fibers of each boundary fibration of $[X ; S]$ to a fiber of a boundary fibration of $X$, and so the composition with $f$ is a resolution fibration.

For ii), first note that $f^{-1}(T)$ is transversal to the fibers of each $\phi_{H}$ for $H \in$ $\mathcal{M}_{1}(X)$ because $f$ is a resolution fibration, and hence $\left[X ; f^{-1}(T)\right]$ has a resolution structure. That the lift of $f$ is a resolution fibration follows as in i).

Finally, for iii), since $\phi_{H}^{-1}(Z)$ is transverse to the fibers of $f$, the composition $\left[X ; \phi_{H}^{-1}(Z)\right] \stackrel{\beta}{\rightarrow} X \stackrel{f}{\rightarrow} M$ is a fibration by Lemma $3.1 \mathrm{i}$ ) and a resolution fibration by the same argument as in i).

Even an equivariant fibration between two manifolds with smooth actions by the same group does not in general lift to a smooth map between their canonical resolutions. However there is a natural resolution of the total space relative to the fibration to which it lifts to fibration to the canonical resolution of the base.

Theorem 9.3. If $f: X \longrightarrow M$ is an equivariant fibration between compact manifolds with smooth actions by a compact Lie group $G$, then there is a natural full resolution of the action on $X$, denoted $Y(X, f)$, such that $f$ lifts to a fibration giving a commutative diagram

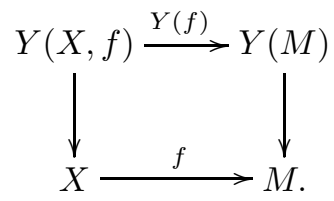

Thus relative resolution of the total space does depend, in general, on the fibration. It reduces to the canonical resolution when the fibration is trivial in the sense that it is either the map to a point or the identity map.

Proof. The first step in the construction of $Y(X, f)$ is to carry out the canonical resolution of $M$ to $Y(M)$. We proceed by induction, assuming both $X$ and $M$ carry resolution structures and that $f$ is an equivariant resolution fibration, as discussed above. The inductive step is to blow up a minimal isotropy type in $M$. The inverse image under the fibration $f$ is a closed p-submanifold of $X$ and Lemma 9.2 ii) shows that after blow of this submanifold $f$ lifts to an equivariant resolution fibration between the resolution structures on the blow-ups of $X$ and $M$. Thus the construction proceeds to give the canonical resolution of $M$ and a total space $X_{0}$, with resolution structure and an equivariant resolution fibration

$$
f_{0}: X_{0} \longrightarrow Y(M)
$$

In general $X_{0}$ is not a partial resolution of $X$ since the base spaces of its boundary fibrations need not have unique isotropy group.

The second part of the procedure is to resolve $X_{0}$, with its resolution structure, without further change to $Y(M)$ and its resolution structure. As in Remark 3.2 $X_{0} \longrightarrow Y(M)$ can also be identified with the pull-back of $X \longrightarrow M$ along $Y(M) \longrightarrow$ $M$. The boundary hypersurfaces of $X_{0}$ are in one-to-one correspondence with the isotropy groups of $M$. Thus for each isotropy type $M_{[K]}$ of $M$, there are boundary 
hypersurfaces $P_{[K]} \subseteq X_{0}$ and $H_{[K]} \subseteq Y(M)$ with fibrations $P_{[K]} \longrightarrow Q_{[K]}$ and $H_{[K]} \longrightarrow Y_{[K]}$ forming a diagram

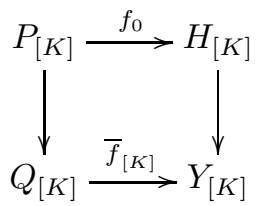

where all arrows are equivariant fibrations. Moreover we may identify $Y_{[K]}$ with $Y\left(M_{[K]}\right)$ as in Remark [7.6 and $\bar{f}_{[K]}: Q_{[K]} \longrightarrow Y_{[K]}$ with the pull-back of $f:$ $f^{-1}\left(M_{[K]}\right) \longrightarrow M_{[K]}$ along the map $Y\left(M_{[K]}\right) \longrightarrow M_{[K]}$.

Lemma 9.4. Suppose $X$ is a manifold with a smooth $G$-action, $Y$ a manifold with a resolved $G$-action and $h: X \longrightarrow Y$ an equivariant fibration then $\left.h\right|_{S}: S \longrightarrow Y$ is surjective for any isotropy type $S \subseteq X$.

Proof. If $\zeta \in S$ then $G_{\zeta}$ is necessarily a subgroup of $K=G_{f(\zeta)}$. If $K$ is a normal subgroup of $G$, then it acts trivially on $Y$, and acts on each fibre of $h$. Proposition 5.5D shows that the fibers of $h$ are $G$-equivariantly diffeomorphic, so an isotropy group that occurs in one fiber occurs in every fiber, and hence $\left.h\right|_{S}$ is surjective.

If $K$ is not a normal subgroup, then the fibration $h$ decomposes into fibrations

$$
h^{K^{\prime}}: h^{-1}\left(Y^{K^{\prime}}\right) \longrightarrow Y^{K^{\prime}}, \quad K^{\prime} \in[K]
$$

and, as each of these is surjective when restricted to $S \cap h^{-1}\left(Y^{K^{\prime}}\right)$, the result follows.

Now, assuming that $X_{0}$ is not already a full resolution of $X$, we proceed to resolve it. Consider all the isotropy types of the action of $G$ on $X_{0}$ and the bases of its boundary fibrations and select one which is minimal (and occurs in an unresolved component of $X_{0}$ or the base of its boundary fibrations). By the discussion in $\$ 7$ it is transverse to the resoution structure and Proposition 3.5 shows that $X_{1}$, obtained by blowing it and all of its preimages in the resolution structure up, has a natural resolution structure. From Lemma 9.2 i) and iii) the composition $X_{1} \longrightarrow X_{0} \longrightarrow Y(M)$ is an equivariant resolution fibration. In case the minimal istropy type occurs in $X_{0}$ itself, it is an interior p-submanifold and the situation is even simpler. In all cases the fibration $f$ lifts to a resolution fibration. Thus, after a finite number of steps the total space and its resolution structure are also resolved.

As for the canonical resolution, the fact that the blow-ups are ordered consistently with the partial order given by inclusion of isotropy groups, and that minimal isotropy types are necessarily disjoint, ensures that this construction of a relative resolution is also natural.

\section{REFERENCES}

[1] P. Albin and R. B. Melrose, Delocalized equivariant cohomology and resolution, forthcoming, see http://arxiv.org/abs/0907.3211

[2] P. Albin and R. B. Melrose, Equivariant families index and resolution, forthcoming.

[3] P. Baum, J.-L. Brylinski, and R. MacPherson, Cohomologie équivariante délocalisée, C. R. Acad. Sci. Paris Sér. I Math. 300 (1985), no. 17, 605-608.

[4] A. Borel and L. Ji, Compactifications of symmetric and locally symmetric spaces, Mathematics: Theory \& Applications, Birkhäuser Boston Inc., Boston, MA, 2006. 
[5] J. Bruening, F. Kamber, and K. Richardson, The eta invariant and equivariant index of transversally elliptic operators, preprint, avaiable online at http://arxiv.org/abs/1005.3845

[6] M. Davis, Smooth G-manifolds as collections of fiber bundles, Pacific J. Math. 77 (1978), no. $2,315-363$.

[7] J. J. Duistermaat and J. A. C. Kolk, Lie groups, Universitext, Springer-Verlag, Berlin, 2000.

[8] A. Hassell, R. Mazzeo, and R. B. Melrose, Analytic surgery and the accumulation of eigenvalues, Comm. Anal. Geom. 3 (1995), no. 1-2, 115-222.

[9] W.-Y. Hsiang, On the classification of differentiable $\mathrm{SO}(n)$ actions on simply connected $\pi$ manifolds, Amer. J. Math. 88 (1966), 137-153.

[10] K. Jänich, On the classification of $O(n)$-manifolds, Math. Ann. 176 (1968), 53-76.

[11] K. Kawakubo, The theory of transformation groups, The Clarendon Press Oxford University Press, New York, 1991.

[12] R. B. Melrose, Differential Analysis on Manifolds with Corners, Unfinished, available online at http://www-math.mit.edu/ rbm/book.html

[13] A. G. Wasserman, Simplifying group actions, Topology Appl. 75 (1997), no. 1, 13-31.

Department of Mathematics, Massachusetts Institute of Technology CURRENT ADDREss: Institut DE Mathématiques De Jussieu

E-mail address: albin@math.jussieu.fr

Department of Mathematics, Massachusetts Institute of Technology

E-mail address: rbm@math.mit.edu 